\title{
Determination of the Correction Factor for Dinalmefene Hydrochloride in Nalmefene Hydrochloride Injection
}

\author{
Tianyi Zhao ${ }^{1}$, Ziqing Liu², Furao Guo ${ }^{1}$, Peiyu He ${ }^{1}$, Hongyu Zhang ${ }^{1}$, Changlong Zhou ${ }^{3}$, Minghua Duan ${ }^{1, *}$ \\ ${ }^{1}$ Changchun University of Chinese Medicine \\ ${ }^{2}$ Changchun University of Technology \\ ${ }^{3}$ Aviation University Air Force
}

\begin{abstract}
Objective: To calculate the detection correction factor for the impurity, that is, the dinalmefene hydrochloride in nalmefene hydrochloride injection. Methods: High performance liquid chromatography (HPLC) is used to analyze the impurities of nalmefene hydrochloride easily produced during storage, and the impurity is determined and correction factor is calculated for the known the dinalmefene hydrochloride. According to the standard curve method, the sample concentration is selected between the detection limit and the limit of quantification, and the standard curve is prepared. The correction factor is then calculated according to the slope of the standard curve. Results: finally, the correction factor for dinalmefene hydrochloride is 0.22 . Conclusions: the correction factor calculated by the standard curve method is accurate and reliable, and can be used for impurity detection of nalmefene hydrochloride injection.

Nalmefene, whose chemical name is 17-cyclopropylmethyl-4, 5-epoxy-6 -methylenemorphinan-3,14-diol, its molecular formula is $\mathrm{C} 21 \mathrm{H} 25 \mathrm{NO} 3$, with the molecular weight of 339.44, and its white powder can be dissolved in water. It was synthesized in 1975 and produced by Miami Pharmaceutical Co., Ltd. of Florida, USA. It is a pure opioid receptor antagonist and is a derivative of water-soluble naltrexone. It can be bound to the opioid receptors $\mu$, $\mathrm{k}$ and $\delta$, and the binding to the $\mu$ receptor is the strongest ${ }^{[1]}$. It can be used for the treatment of natural or synthetic narcotic analgesic poisoning, and also has certain antagonistic effects on some non-opioid poisoning ${ }^{[2]}$.

Since nalmefene hydrochloride is easy to produce impurities and is unstable during storage, this experiment attempts to determine the impurity correction factor for impurities easily produced by nalmefene hydrochloride.
\end{abstract}

\section{Experimental materials}

HPLC (LC-15C) (Shimadzu, Japan), C18 $(150 \times 4.6 \mathrm{~mm}$, $5 \mu \mathrm{m})$ (Elite, Dalian), acetonitrile (Fisher, USA), sodium dihydrogen phosphate, triethylamine (Beijing Chemical Plant, Beijing).

\section{Experimental methods}

\subsection{Chromatographic conditions}

Mobile phase: acetonitrile-phosphate buffer (take $7.8 \mathrm{~g}$ of sodium dihydrogen phosphate, add $2 \mathrm{ml}$ of triethylamine with water to $1000 \mathrm{ml}$, adjust the $\mathrm{pH}$ to $4.2 \pm 0.02$ with $85 \%$ phosphoric acid) (20:80); Detection wavelength: $210 \mathrm{~nm}$; flow rate: $1.0 \mathrm{ml} / \mathrm{min}^{[3]}$

\subsection{Establishment of detection wavelength}

The TU-1810 ultraviolet-visible spectrophotometer was used to perform full-wavelength scanning of nalmefene hydrochloride injection to determine its maximum absorption.

\subsection{Determination of quantitative limit and repeatability detection of nalmefene hydrochloride}

Take this product $(0.1 \mathrm{mg} / \mathrm{ml})$ as a test solution, compare the signal of known low concentration sample with that of blank sample, and then calculate the lowest concentration or quantity that can be reliably detected. In general, the limit of quantitation is determined by the corresponding concentration at a signal to noise ratio of 10:1 or the amount of instrument injected. The measurement was repeated 3 times according to the determined limit of quantitation of the substance concerned.

\subsection{Determination of the limit of quantitation and repeatability determination of dinalmefene hydrochloride ${ }^{[4]}$}

\footnotetext{
${ }^{*}$ Corresponding author: Duan Minghua, duanduan-2007@163.com
} 
A solution of dinalmedefene hydrochloride $(1.0 \mu \mathrm{g} / \mathrm{ml})$ was used as a test solution to determine the limit of quantitation. The measurement was repeated 3 times according to the determined limit of quantitation of the substance concerned.

\subsection{Determination of limit and repeatability detection of nalmefene hydrochloride}

Take a quantitative limit concentration sample (300 $\mathrm{ng} / \mathrm{ml}$ ) as a test solution, compare the signal measured by the known low concentration sample with the signal measured by the blank sample, and calculate the lowest concentration or amount that can be reliably detected. The detection limit is generally determined by the corresponding concentration at a signal to noise ratio of $3: 1$ or the amount of instrument injected.

\subsection{Determination of detection limit and repeatability determination of dinalmefene hydrochloride}

A solution of diamedefine hydrochloride $(300 \mathrm{ng} / \mathrm{ml})$ was taken as a test solution, to measure the detection limit. The measurement was repeated 3 times according to the determined detection limit of the relevant substance.

\subsection{Preparation of nalmefene hydrochloride solution}

According to the limit of quantitation of nalmefene hydrochloride, solutions with five concentrations of 1200 $\mathrm{ng} / \mathrm{ml}, 600 \mathrm{ng} / \mathrm{ml}, 300 \mathrm{ng} / \mathrm{ml}, 150 \mathrm{ng} / \mathrm{ml}$, and $75 \mathrm{ng} / \mathrm{ml}$ were prepared respectively.

\subsection{Preparation of diamedefine hydrochloride solution}

According to the detection limit and limit of quantitation of dinalmefene hydrochloride, five solutions with concentrations of $1200 \mathrm{ng} / \mathrm{ml}, 600 \mathrm{ng} / \mathrm{ml}, 300 \mathrm{ng} / \mathrm{ml}, 150$ $\mathrm{ng} / \mathrm{ml}$, and $75 \mathrm{ng} / \mathrm{ml}$ were prepared respectively.

\section{Experimental results}

\subsection{Establishment of detection wavelength}

A full-wavelength scan of nalmefene hydrochloride was performed by using an ultraviolet-visible spectrophotometer (TU-1810 UV-Vis spectrophotometer) to determine its maximum absorption, as shown in Figure 1 below.

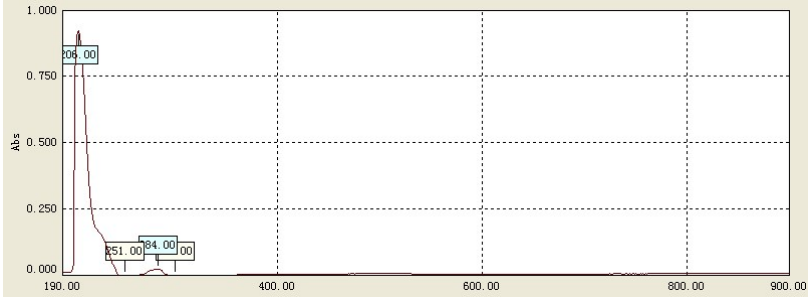

Figure.1 Ultraviolet scanning spectrum of nalmefene hydrochloride solution.

As can be seen from the above figure, this product has maximum absorption at $206 \mathrm{~nm}$. The detection wavelength of the substance related to nalmefene hydrochloride injection is $210 \mathrm{~nm}$, so $210 \mathrm{~nm}$ is suitable for the determination of related substances in this product.

\subsection{Determination of quantitative limit and repeatability detection of nalmefene hydrochloride}

Precisely measure the appropriate amount of test solution. According to the literature and empirical values, finalize the 333-times dilution with water to obtain $300 \mathrm{ng} / \mathrm{ml}$ test solution. The ratio of peak height and noise peak height of nalmefene component is determined to be 10.1:1 according to the proposed method. Therefore, the final quantitative limit of nalmefene was determined to be 30 ng, as shown in Figure 2A.

According to the determined limit of quantification of the relevant substances, the concentration of $300 \mathrm{ng} / \mathrm{ml}$ nalmefene hydrochloride injection was prepared and repeated the procedure 3 times. The test results are shown in Table 1 below.

Table 1. Repeatability of quantitative limit concentration test for related substances in nalmefene hydrochloride.

\begin{tabular}{|c|c|c|}
\hline Serial No. & Signal-to-noise ratio & $\operatorname{RSD}(\%)$ \\
\hline 1 & $10.1: 1$ & \multirow{2}{*}{0.57} \\
\hline 2 & $10.2: 1$ & \\
\hline 3 & $10.1: 1$ & \\
\hline
\end{tabular}

The test results show that the standard deviation between the relevant substance samples with quantitative limit concentration and the test results is consistent with the requirements, indicating that the determined limit of quantitation of the nalmefene is accurate and reliable.

\subsection{Determination of quantitative limit and repeatability detection of dinalmefene hydrochloride}

Accurately measure the appropriate amount of test solution, finalize the 333-times dilution with water to obtain $300 \mathrm{ng} / \mathrm{ml}$ test solution according to the literature and empirical values. The ratio of the peak height to noise peak of nalmefene component was determined to be 10:1 according to your method. Therefore, the quantitative limit of dinalmefene was determined to be 30 ng, as shown in Figure 2C. 
According to the quantitative limit of the related substances, the concentration of dinamefen hydrochloride solution was $300 \mathrm{ng} / \mathrm{ml}$. The procedures were repeated for three times. The results were shown in Table 2 below.

Table 2. Reproducibility of concentration limit test of dinamefen hydrochloride.

\begin{tabular}{|c|c|c|}
\hline Serial No. & Signal-to-noise ratio & \multirow{2}{*}{$\operatorname{RSD}(\%)$} \\
\hline 1 & $10: 1$ & \multirow{2}{*}{0.57} \\
\hline 2 & $10.1: 1$ & \\
\hline 3 & $10: 1$ & \\
\hline
\end{tabular}

The test results show that the standard deviation between the test results and the sample of the quantitative limit concentration are in accordance with the regulations, which indicates that the determined quantitative limit of dinalmefene hydrochloride is accurate and reliable.

\subsection{Determination of detection limit and repeatability detection of nalmefene hydrochloride}

Precisely measure the appropriate amount of test solution. According to the literature and empirical values, the water is finally determined to be diluted 4 times to obtain $75 \mathrm{ng} / \mathrm{ml}$ test solution. According to the [related substances] method under the quality standard of nalmefene hydrochloride injection, the high performance liquid chromatograph was used to measure the relevant substances in the above test solution, and the ratio of peak height to noise peak height of the nalmefene component was 3.1:1. Therefore, the detection limit of nalmefene was $7.5 \mathrm{ng}$, as shown in Figure 2B.

According to the determined detection limit of the related substances, the concentration of nalmefene hydrochloride injection was $75 \mathrm{ng} / \mathrm{ml}$. The procedures were repeated for three times. The results were shown in Table 3.

Table 3. Repeatability of concentration test of detection limit for related substances in nalmefene hydrochloride.

\begin{tabular}{|c|c|c|}
\hline Serial No. & Signal-to-noise ratio & \multirow{2}{*}{$\operatorname{RSD}(\%)$} \\
\hline 1 & $3.1: 1$ & \multirow{2}{*}{1.88} \\
\hline 2 & $3.1: 1$ & \\
\hline 3 & $3.0: 1$ & \\
\hline
\end{tabular}

The test results show that the standard deviation between the relevant substance samples with quantitative limit concentration and the test results is consistent with the requirements, indicating that the determined nalmefene detection limit is accurate and reliable.

\subsection{Determination of detection limit and repeatability detection of dinalmefene hydrochloride}

Accurately measure the appropriate amount of test solution, according to the literature and empirical values, finally determine the addition of water for 4 times dilution, get $75 \mathrm{ng} / \mathrm{ml}$ test solution. The ratio of the peak height to noise peak of nalmefene component is determined to be 3.1:1 according to your method.
Therefore, the detection limit of nalmefene was finally determined to be $7.5 \mathrm{ng}$, as shown in Fig. 2D.

According to the determined detection limit of the relevant substances, a solution of $75 \mathrm{mg} / \mathrm{ml}$ dinalmefene hydrochloride solution of the nalmefene hydrochloride was prepared and the procedures were repeated 3 times. The test results were shown in Table 4 below.

Table 4. Repeatability of concentration test of detection limit in dinalmefene hydrochloride.

\begin{tabular}{|c|c|c|}
\hline Serial No. & Signal-to-noise ratio & RSD $(\%)$ \\
\hline 1 & $3.1: 1$ & \multirow{2}{*}{1.88} \\
\hline 2 & $3.0: 1$ & \\
\hline 3 & $3.1: 1$ & \\
\hline
\end{tabular}

The test results show that the standard deviation between the relevant substance samples with quantitative limit concentration and the test results is consistent with the requirements, which indicates that the determined detection limit of dinalmefene in nalmefene hydrochloride is accurate and reliable.

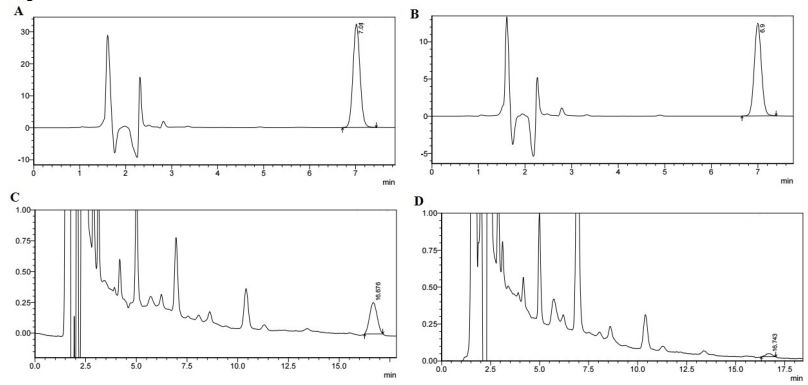

Figure 2. Quantitative limit and detection limit.

\subsection{Standard curve drawing of correction factor detection for nalmefene hydrochloride}

The nalmefene hydrochloride solutions with five concentrations were determined according to the [related substances] detection method, and the results were shown in Table 5 below.

Table 5. standard curve drawing for determining correction factor for nalmefene hydrochloride.

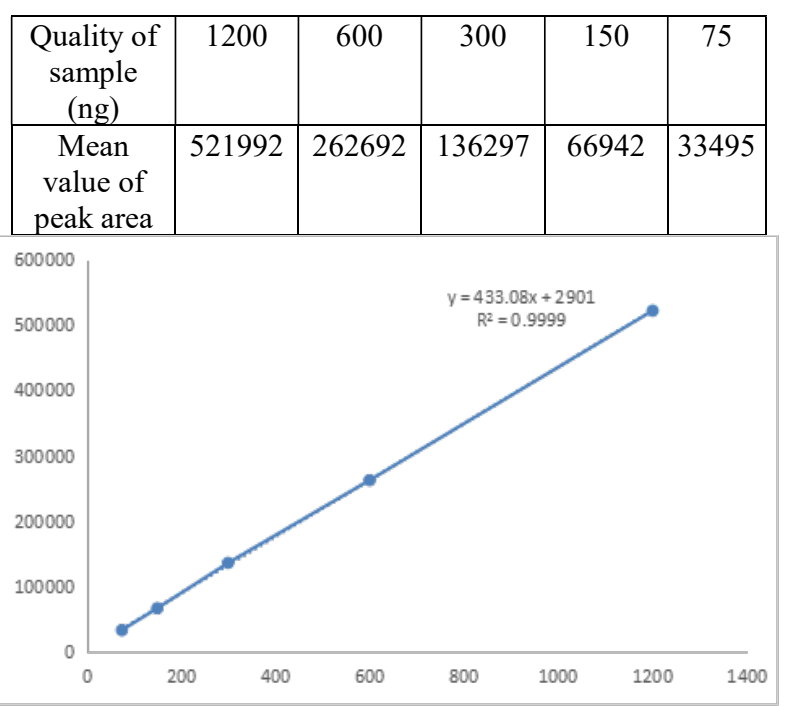

Figure 3. Standard curve of correction factor for nalmefene hydrochloride. 


\subsection{Standard curve drawing for determining correction factor for dinalmefene hydrochloride}

The five dinalmefene hydrochloride solutions with different concentrations were measured according to the [related substances] detection method, and the measurement results are shown in Table 6 below.

Table 6. Standard curve drawing for determining correction factor for dinalmefene hydrochloride.

\begin{tabular}{|c|c|c|c|c|c|}
\hline $\begin{array}{c}\text { Quality } \\
\text { of sample } \\
\text { (ng) }\end{array}$ & 120 & 60 & 30 & 15 & 7.5 \\
\hline $\begin{array}{c}\text { Peak area } \\
1\end{array}$ & 231739 & 114610 & 54912 & 25927 & 12846 \\
\hline $\begin{array}{c}\text { Peak area } \\
2\end{array}$ & 231263 & 111261 & 56284 & 23675 & 10156 \\
\hline $\begin{array}{c}\text { Peak area } \\
3\end{array}$ & 231750 & 112177 & 53345 & 24199 & 10249 \\
\hline $\begin{array}{c}\text { Mean } \\
\text { value }\end{array}$ & 231584 & 112683 & 54607 & 24600 & 11084 \\
\hline $\begin{array}{c}250000 \\
200000\end{array}$ \\
150000
\end{tabular}

Figure 4. Standard curve of correction factor for dinalmefene hydrochloride.

\subsection{Calculation of the correction factor for dinalmefene hydrochloride[5]}

The slope of the standard curve of nalmefene hydrochloride was 433.08, and the slope of the standard curve of dinalmefene hydrochloride was 1963.5 , and the ratio of them was 0.22 . Therefore, the correction factor for dinalmefene hydrochloride in hydrochloride was 0.22 .

\section{Discussion}

Nalmefene hydrochloride is mainly applied in the antagonism of narcotic analgesic respiratory depression, heart failure and shock treatment, alcoholism and alcohol addiction. It is the latest generation of opioid receptor antagonists for neuroprotective treatment, and it is the first choice for acute, moderate to severe spinal cord, respiratory depression first aid and awakening in acute moderate to severe spinal cord and brain injury. The drug is of quick, long-lasting and safe effect.

Nalmefene injection (REVEX) was developed by Ohmeda Pharmaceutical Company. It was approved by
FDA on April 17, 1995 and is the only dosage form on the market at present. The preparation has the physiological saline solutions ( $\mathrm{pH}$ 3.9) with two specifications of $0.1 \mathrm{~g} / \mathrm{L}$ and $1 \mathrm{~g} / \mathrm{L}$. The product was discontinued in 2008. At present, there are only four kinds of nalmefene hydrochloride injections sold in the market in China, all of which are domestically produced products, and the products are only valid for 12 months.

The reason for the above situation is that the nalmefene hydrochloride injection is unstable, and the dimer content is increased during storage, resulting in a decline in product quality until it is unqualified. Many scholars and experts are studying ways to improve stability of nalmefene hydrochloride injection.

Based on the study of the quality standard of nalmefene hydrochloride injection, this paper formulated the correction factor for the detecting nalmefene hydrochloride injection [related substances] ${ }^{[6]}$. In order to make the peak area accurately reflect the content of the component to be tested, it is needed to determine the peak area under the chromatographic conditions by using a known amount of the component to be tested, so as to calculate the correction factor. The impurity content calculated by the correction factor is more accurate and the quality of the product is more reliable.

\section{References}

1. Liu Xing-hua, Chen Chun-you, Zheng Lin et al. Efficacy of nalmefen hydrochloride and effect on serum IL-8, NSE and S100 $\beta$ levels in acute craniocerebral injury. Chinese Journal of Biochemistry and Pharmacy, 2016, 7(36):97-99

2. Wang Yuan-wen, Zhao Wen-lin, Li Qing. Effect of nalmefene hydrochloride adjuvant therapy on serum $\mathrm{NO}$, endothelin and cardiac troponin I in patients with traumatic shock. Chinese Journal of Biochemistry and Pharmacy, 2015, 11(35):149-151

3. Zhong $\mathrm{Wu}$, Zheng Zhi-bin, Xiao Jun-hai et al. HPLC determination of nalmefene hydrochloride and its related substances.Chinese Jouranl of New Drugs, 2006, 15(11):895-898

4. Yao Wen-jing, Li Wan-qing, Sun Jian-xu et al. Determination of disodium edetate in nalmefene hydrochloride injection by HPLC.J Int Pharm Res, 2017, 44(1):70-73

5. Rem Chun, Wu Yang, Zhang Di et al. Determination of Impurities in Pantoprazole Sodium. Pharm J Chin PLA, 2012, 28(4):323-330

6. Ding Rui, Ji Hong, Cheng Si et al. Assay of the related substances in alprostadil for injection with the correction factor. Journal of China Pharmaceutical University, 2010, 41(5):462-466 